\title{
Distinct inter-joint coordination during fast alternate keystrokes in pianists with superior skill
}

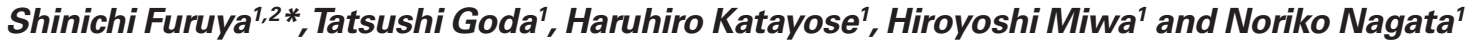 \\ School of Science and Technology, Kwansei Gakuin University, Hyogo, Japan \\ 2 Research Fellow, Japan Society for the Promotion of Science, Tokyo, Japan
}

\section{Edited by:}

Robert J. Zatorre, McGill University,

Canada

\section{Reviewed by:}

Peter Keller, Max Planck Institute for

Human Cognitive and Brain Sciences, Germany

Virginia Penhune, Concordia University, Canada

Caroline Palmer, McGill University,

Canada

* Correspondence:

Shinichi Furuya, School of Science and Technology, Kwansei Gakuin University, 2-1, Gakuen, Sanda, Hyogo 669-1337, Japan.

e-mail: auditory.motor@gmail.com
Musical performance requires motor skills to coordinate the movements of multiple joints in the hand and arm over a wide range of tempi. However, it is unclear whether the coordination of movement across joints would differ for musicians with different skill levels and how interjoint coordination would vary in relation to music tempo. The present study addresses these issues by examining the kinematics and muscular activity of the hand and arm movements of professional and amateur pianists who strike two keys alternately with the thumb and little finger at various tempi. The professionals produced a smaller flexion velocity at the thumb and little finger and greater elbow pronation and supination velocity than did the amateurs. The experts also showed smaller extension angles at the metacarpo-phalangeal joint of the index and middle fingers, which were not being used to strike the keys. Furthermore, muscular activity in the extrinsic finger muscles was smaller for the experts than for the amateurs. These findings indicate that pianists with superior skill reduce the finger muscle load during keystrokes by taking advantage of differences in proximal joint motion and hand postural configuration. With an increase in tempo, the experts showed larger and smaller increases in elbow velocity and finger muscle co-activation, respectively, compared to the amateurs, highlighting skill leveldependent differences in movement strategies for tempo adjustment. Finally, when striking as fast as possible, individual differences in the striking tempo among players were explained by their elbow velocities but not by their digit velocities. These findings suggest that pianists who are capable of faster keystrokes benefit more from proximal joint motion than do pianists who are not capable of faster keystrokes. The distinct movement strategy for tempo adjustment in pianists with superior skill would therefore ensure a wider range of musical expression.

Keywords: multi-joint movements, postural control, stiffness control, long-term training, musicians, music, hand-arm coordination, musical expression

\section{INTRODUCTION}

Artistic musical performance involves variation of multifaceted aspects of music. Precise manipulation of musical components, a skill necessary for evoking intended emotional responses to audience, requires dexterous control of hand movements and postures that is coordinated with arm movements. Studying musical performance therefore provides a good opportunity to probe into how the nervous system skillfully orchestrates a redundant number of degrees of freedom (DOFs) of the motor system to achieve artistic musical expression. Previous studies have extensively investigated repetitive hand movements during musical performance or more simplified tasks (Parlitz et al., 1998; Aoki et al., 2005; Goebl and Palmer, 2008; Fujii et al., 2009a,b; Loehr and Palmer, 2009; Palmer et al., 2009; Furuya and Soechting, 2010). Some of these studies have delineated differences in the characteristics of force exerted by digits (Parlitz et al., 1998; Aoki et al., 2005) and in the activities of extrinsic finger muscles (Fujii et al., 2009a,b) between musicians and non-musicians. For example, Parlitz et al. (1998) measured the force applied to the keys while professional and amateur piano players alternately struck two keys. They found that the professionals showed shorter durations of force application to the keys after the keys reached their bottom position than did the amateurs, indicating a reduction in unnecessary exertion of force. Fujii et al. (2009a) found that highly skilled drummers exhibited less tonic and more reciprocal activity at the finger muscles during finger tapping compared with non-drummer controls. These findings commonly suggested lower energetic cost of movements by musicians with superior skill. However, neither movement organization reflecting superior skill nor its relation to movement rate (tempo) has been addressed. To identify them provides insight into neural control of artistic musical performance, because manipulation of music tempo, which elicits emotional and autonomic responses to individuals listening to music (Dalla Bella et al., 2001; Khalfa et al., 2008), is essential for a wide variety of musical expressions.

Bernstein (1967) postulated that improvements in motor skills would be associated with the use of a greater number of DOFs to economize movement production. For example, involvement of additional joint in movement production can generate the intersegmental dynamics that propel motions at the adjacent joints in place of muscular force (Dounskaia, 2010). In agreement with this postulation, recent studies have demonstrated that skilled pianists reduced muscular work while striking a key by utilizing a greater number of DOFs compared with novice piano players (Furuya and Kinoshita, 2007, 2008a,b; Furuya et al., 2009). These results showed 
that compared with novices, professional pianists decelerated their shoulder extension to a greater degree during arm downswing to generate larger inter-segmental dynamics that accelerate motion at the elbow and wrist joints, thereby reducing the distal muscular load. The pianists also rotated their shoulder for flexion to a greater extent than the novices while depressing keys, thereby reducing the finger muscular load to compensate for the key reaction force. These findings indicate that individuals with superior piano skills economize the work performed by distal muscles during a piano keystroke by taking advantage of proximal joint motion. One limitation of these studies was that they focused on a discrete keystroke motion. Due to distinct differences in the control mechanism underlying discrete and cyclic movements (Schaal et al., 2004; Hogan and Sternad, 2007; Huys et al., 2008), it is worthwhile to probe whether the skill level-dependent difference that has been observed for a discrete piano keystroke is also present for cyclic keystrokes.

Another key issue in musical performance is how to change the organization of movement to modulate tempo. A small number of studies have investigated the effect of tempo on the upper-limb movements while pianists were striking two keys simultaneously and repetitively (Kay et al., 2003; Furuya et al., 2011). The results of these studies have shown that as the tempo increased, professional pianists changed joint velocity in a non-uniform manner; for example, increased their shoulder and wrist velocity and decreased their elbow velocity (Furuya et al., 2011). This differential effect of tempo variation on movements across joints has also been observed in studies investigating cyclic arm movements during a handdrawing task (Meulenbroek et al., 1993; Pfann et al., 2002). For example, a study of a circle-drawing task found that as movement tempo increases, a majority of subjects use more elbow motion than shoulder motion (Pfann et al., 2002). However, it is unclear whether variation of inter-joint coordination in relation to tempo could differ for individuals with different skill levels.

The present study aimed to address the effect of tempo on the organization of hand and forearm movements and finger muscular activity while both professional and amateur piano players struck two piano keys alternately with the thumb and little finger. This motor task, referred to as "tremolo," was chosen because the movement is performed cyclically in three-dimensional space, requiring a larger number of DOFs to be controlled for both movement production and postural maintenance compared with the planar movements that have been primarily studied previously. We hypothesized that inter-joint coordination of movements during repetitive keystrokes would vary in relation to both tempo and skill level. Specifically, we postulated that pianists with superior skill would utilize proximal joint motion to a greater extent to strike keys, keep non-striking fingers less extended, and lessen finger muscular load during keystrokes and that these skill level-dependent differences would become more pronounced at faster tempi.

The present study also probed into the movement features associated with individual differences in the fastest striking tempo for piano players. Extremely fast hand movements represent the motor skills of virtuoso musicians, and their underlying neural mechanisms, including gray matter volume of motor cortex (Amunts et al., 1997; Münte et al., 2002) and cerebellum (Hutchinson et al., 2003) and white matter volume (Bengtsson et al., 2005), have been studied extensively. Behavioral studies using finger tapping have also illustrated superior hand motor functions of musicians (Jäncke et al., 1997; Aoki et al., 2005). Nevertheless, an understanding of the movement characteristics that enable extremely fast cyclic limb motion during musical performance remains elusive.

\section{MATERIALS AND METHODS PARTICIPANTS}

Five active expert pianists ("professional;" two males and three females, mean age $\pm S D=24.3 \pm 3.2$ years, all right-handed) with more than 20 years of formal classical piano training and five recreational pianists who have no history of professional music education but practiced for less than $3 \mathrm{~h}$ per week ("amateur;" three males and two females, mean age $\pm \mathrm{SD}=22.6 \pm 1.1$ years, all right-handed) participated in the present study. All of the professional pianists had won awards at domestic and/or international classical piano competitions. The group mean of the hand span was 203.6 \pm 15.1 and $195.2 \pm 15.1 \mathrm{~mm}$ for the professionals and amateurs, respectively ( $t$-test: $p=0.55$ ). The group mean of the body mass was $54.4 \pm 13.6$ and $55.8 \pm 9.8 \mathrm{~kg}$ for the professionals and amateurs, respectively ( $t$-test: $p=0.88$ ). A lack of significant group difference in both of these variables confirmed that their anthropometry did not account for any observed differences in kinematics and muscular activities between the two groups. In accordance with the Declaration of Helsinki, the experimental procedure was clearly explained to the participants, who submitted written informed consent. The study was approved by the local ethics committee at Kwansei Gakuin University.

\section{EXPERIMENTAL APPARATUS AND KEY-STRIKING TASK}

The experimental apparatus used was a digital piano with a touch response action (P-250 YAMAHA Co.), a motion-capturing system consisting of 13 high-speed cameras (eight Eagle and five Hawk Eye, Mac3D system, Motion Analysis Co.), and a two-channel electromyography (EMG) system (Harada Electronics Industry Ltd.; Figure 1A). To collect positional data on anatomical landmarks, spherical reflective markers $(5 \mathrm{~mm}$ in diameter for the hand and key and $9 \mathrm{~mm}$ in diameter for the wrist and elbow) were attached to two separate keys and on all joint centers of the right hand and arm. The experimental task was to perform repetitive tremolo keystrokes with the right hand, alternating keystrokes of the 52nd key (E) by the thumb and the 60th key (C) by the little finger (Figure 1B). The keys were $118 \mathrm{~mm}$ apart. This motor task (tremolo) is widely used in a variety of musical pieces written by composers such as Beethoven, Chopin, Schumann, Liszt, and so on. The findings derived from the present study should thus provide information that would be useful for pianists and piano teachers. Before starting the keystroke task, a striking tempo was provided to the participant by a metronome for $5 \mathrm{~s}$. Then, the participant was cued to start striking the keys. Each trial consisted of keystrokes for 10 s. During each trial, the metronome continued to provide the tempo. The left arm and hand were kept relaxed on the side of the body while the trunk and right upper arm were placed in an upright position with minimal movement.

Five target striking tempi of 70, 90, 110, 130, and $260 \mathrm{bpm}$ were chosen in this study, which correspond to $857,667,545,462$, and $231 \mathrm{~ms}$, respectively, of inter-keystroke intervals (IKI) of one key (i.e., two successive strokes of the E key). Hence, the expected IKI 


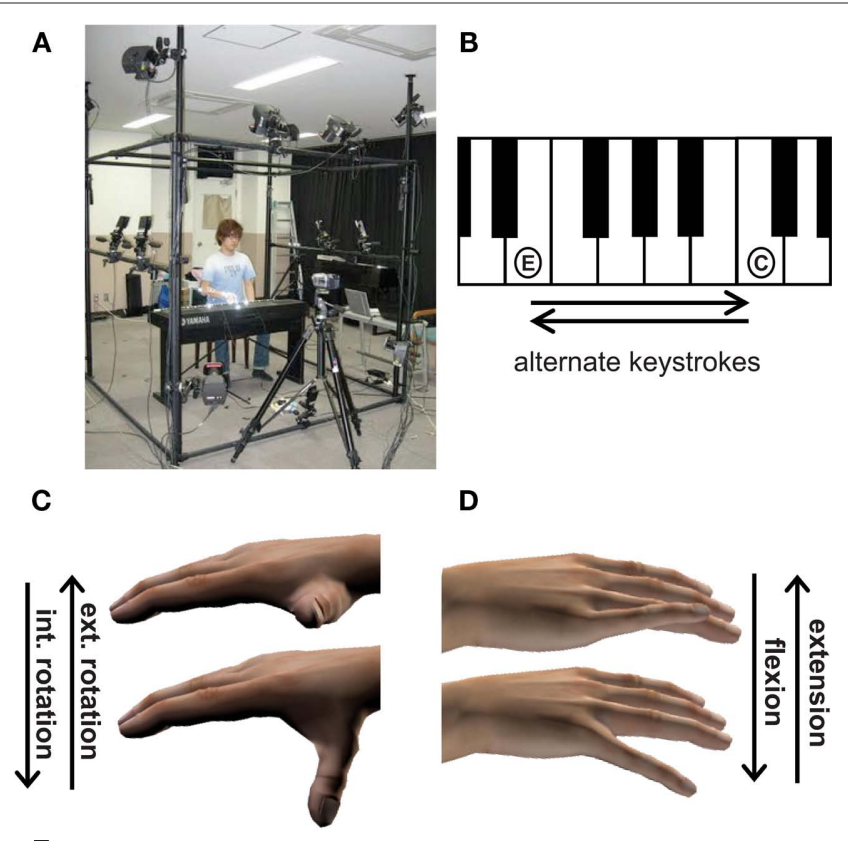

E

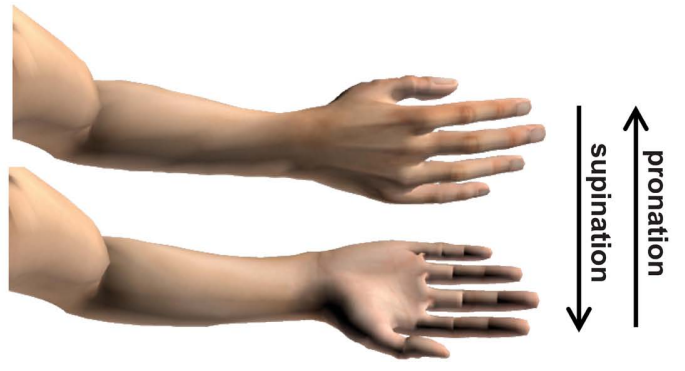

FIGURE 1 | (A) Experimental setup. (B) Experimental task. The E and $C$ keys were struck alternately with the thumb and little finger, respectively. (C-E) Thumb internal/external rotation (C), little finger MCP flexion/extension (D), and elbow pronation/supination rotation (E).

between successive strokes of two different keys (e.g., from E stroke to the following $\mathrm{C}$ stroke) was half of them, ranging from 428.5 to $115.5 \mathrm{~ms}$ for these five tempi. Each participant was also asked to perform the designated task as fast as possible. We did not provide participants with any instruction regarding a manner to depress the keys ("touch"). The target loudness for the tone was set to approximately 65 MIDI velocity during the task and was monitored by an experimenter during each trial. The loudness was set to a constant level because our previous study showed an significant interaction effect of loudness and tempo on the upper-limb movements during repetitive piano keystrokes (Furuya et al., 2011).

\section{DATA ACQUISITION PROCEDURES}

Twenty-five spherical reflective markers were mounted on the keys and the body to identify anatomical landmarks for the purpose of digitalization. These markers were placed on the skin over the fingertips and three joint centers of all five digits, the distal end of the radius, the proximal and distal ends of the ulna, and the two piano keys. The motion of the reflective markers was recorded at $120 \mathrm{~Hz}$ using 13 high-speed cameras surrounding the piano (Figure 1A). The camera locations were carefully arranged so that one could continue to record position data of all of the markers while performing the target task. The spatial resolution in the camera setting was $1 \mathrm{~mm}$. The 3D time-position data of each marker was obtained by the direct linear transformation method. All procedures were established in a previous study, which was conducted for the purpose of creating CG animations based on motion-capturing data derived while playing the piano (Kugimoto et al., 2009). The data were digitally smoothed at a low-pass cutoff frequency of $15 \mathrm{~Hz}$ using a second-order Butterworth digital filter. Subsequently, the following joint angles were computed: elbow pronation/supination rotation about an axis passing through the proximal and distal ends of the ulna, thumb internal/external rotation about an axis passing through the trapeziometacarpal joint of the thumb and the index metacarpo-phalangeal (MCP) joint, MCP, and proximalphalangeal (PIP) flexion/extension rotation at the index, middle, and ring fingers, and MCP flexion/extension rotation at the little finger (Figures 1C-E). These computations were based on procedures proposed previously (Feltner and Taylor, 1997; Hirashima et al., 2007). We did not use data regarding angles at the IP and MCP joints at the thumb and little PIP joints for subsequent analysis because our pretest of two professionals and two amateurs found that the rotational motion at these joints was indiscernible in the present task.

The electrical activity of the right side of the extensor digitorum communis (EDC) and flexor digitorum superficialis (FDS) muscles was recorded with the surface EMG system. In our pretest, we also attempted to record the activity at the forearm muscles responsible for the pronation and supination rotations (supinator and pronator teres muscles), but our surface EMG system failed to do it reliably due to substantial cross-talk from adjacent muscles. Pairs of $\mathrm{Ag} / \mathrm{AgCl}$ surface disposal electrodes were placed at the estimated center of each target muscle with a 20 -mm center-to-center difference. The electrode position was carefully determined to minimize cross-talk from adjacent muscles. At each electrode position, the skin was shaved, abraded, and cleaned using isopropyl alcohol to reduce source impedance. The EMG signals were amplified 5000-fold and sampled at $960 \mathrm{~Hz}$ using an A/D converter interfaced with a personal computer. The signals were then digitally high-pass filtered (with a cutoff frequency of $20 \mathrm{~Hz}$ ) to remove movement artifacts and then root-mean squared. To normalize these EMG data for each muscle in each participant, EMG data during maximum voluntary contraction (MVC) were obtained for each muscle by asking the participant to perform maximum flexion or extension with an isometric force against a stationary object for a 5-s period. Each participant was verbally encouraged to achieve maximal force at a designated joint angle. During an MVC trial for the EDC and FDS muscles, the finger and wrist joints were kept at $180^{\circ}$. A percentage of the MVC value was then calculated using the mean value of the middle 3-s period of the MVC data. To confirm that each participant exerted maximum force, the MVC trial was repeated twice for each muscle, and the mean MVC value of these two trials was computed. Due to a lack of any apparent difference between the two MVC values ( $t$-test: $p=0.69$ ), we simply computed the mean rather than the highest value.

\section{DATA ANALYSIS}

The onset of descending movement for both the $\mathrm{E}$ and the $\mathrm{C}$ keys ("finger-key contact moment") was determined when the computed vertical velocity of the key exceeded $5 \%$ of its peak velocity. 
During each time window between two successive strokes of the $\mathrm{E}$ key (i.e., one IKI), we computed a set of kinematic and EMG variables, which were then averaged across all IKI during each trial. The following kinematic variables were computed: (1) peak values of both the thumb internal rotation velocity and the MCP flexion velocity of the little finger, (2) peak values of the supination and pronation angular velocity at the elbow joint, and (3) mean angle of the MCP and PIP joints at the index, middle, and ring fingers (i.e., non-striking fingers) during an IKI. To eliminate the background noise in the EMG variables, we initially subtracted the mean value of the muscular activity recorded while the hand and forearm were kept relaxed on the table. We then computed the mean values of the activities of the EDC and FDS muscles. In addition, we used the following equation to compute the co-activation index (CI) based on previous studies (Kellis et al., 2003; Furuya and Kinoshita, 2008b; Furuya et al., 2011) as an estimate of joint stiffness:

$\mathrm{CI}=\frac{\left(\int_{t 1}^{t 2} \mathrm{EMG}_{\text {agon }}(t) d t+\int_{t 2}^{t 3} \mathrm{EMG}_{\text {ant }}(t) d t\right)}{\Delta T}$

where the period from $t 1$ to $t 2$ denotes the time when the agonist EMG activity is less than the antagonist EMG and vice versa for the period from $t 2$ to $t 3 . \Delta T$ is the IKI.

\section{STATISTICS}

Using group (professional/amateur) and tempo (five predetermined tempi) as independent variables, a two-way analysis of variance (ANOVA) with repeated measurements was performed for each of the dependent variables. Newman-Keuls post hoc tests were performed where appropriate to correct for multiple comparisons. Statistical significance was set at $p<0.05$.

\section{RESULTS}

\section{KEY-STRIKING VELOCITY AND INTER-KEYSTROKE INTERVAL}

Table 1 shows the mean MIDI velocity and IKI of the E and C keys across participants in the two groups at different tempi. Two-way repeated measures ANOVA demonstrated no difference in MIDI velocity for different tempi and between the two groups, confirming tone volume consistency. The IKI was systematically decreased in proportion to tempo for both groups, all of which followed the IKI designated by the metronome $(857,667,545,462$, and $231 \mathrm{~ms}$ for 70, 90, 110, 130, and $260 \mathrm{bpm}$, respectively). These findings confirmed that both professional and amateur pianists successfully performed the designated task.

\section{PROFILE OF JOINT ANGULAR VELOCITY}

Figure 2 illustrates the profiles of the joint angular velocity of the elbow, little finger, and thumb across different tempi $(70,130$, and $260 \mathrm{bpm}$ ) for one representative professional and one representative amateur pianist. The results show that the angular velocity for elbow supination and pronation, little MCP flexion, and thumb internal rotation reached their peaks prior to the keypress moment for both players. In addition, the timing of peak velocity occurred earlier for both players as tempo increased. The professional pianist showed greater peak angular velocity for the elbow and smaller peak velocity for the thumb and little finger for all three tempi compared to the amateur pianist.

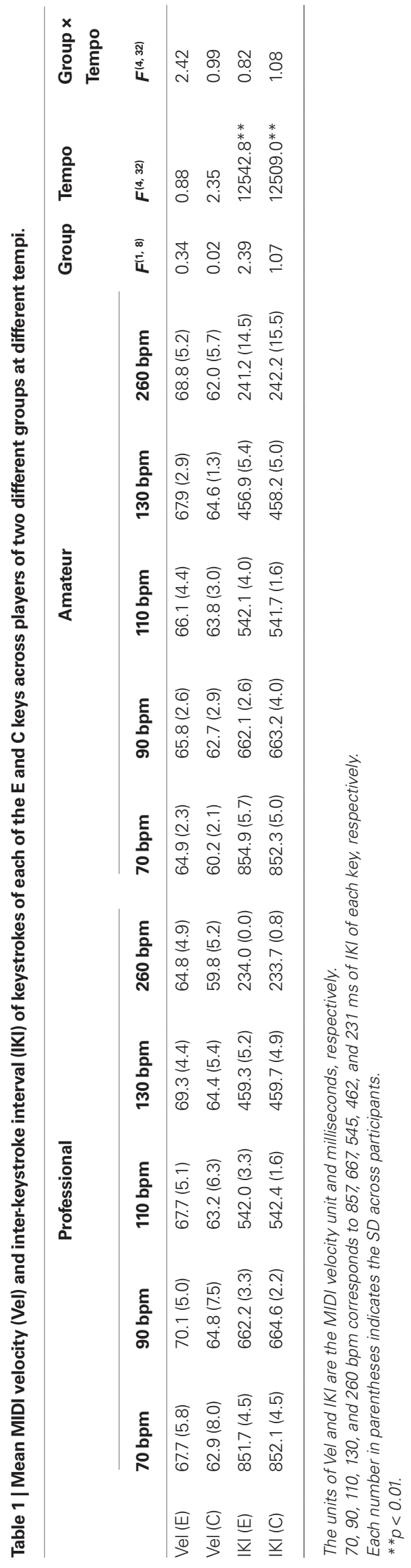




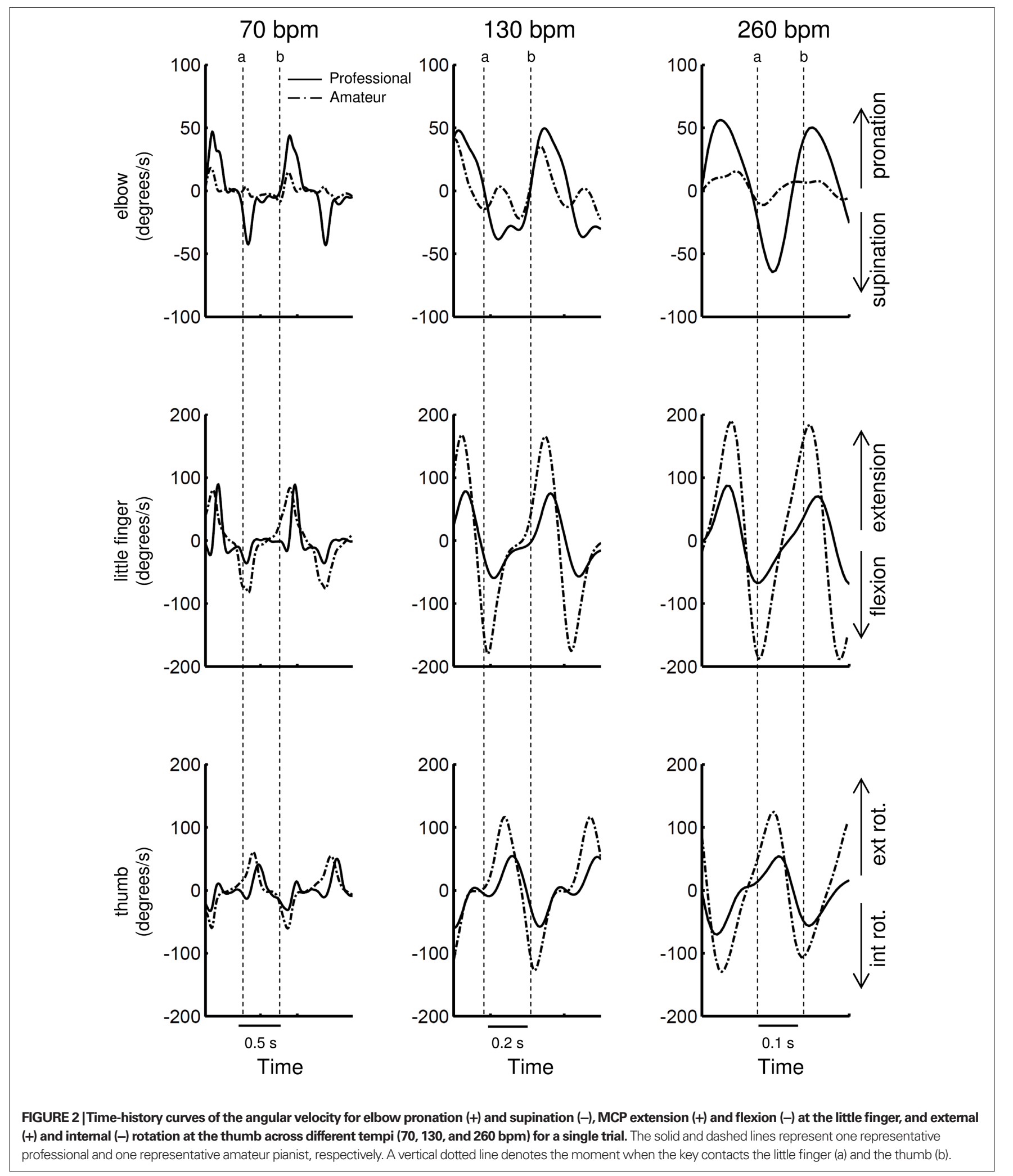

PEAK ANGULAR VELOCITY AT THE ELBOW, LITTLE FINGER, AND THUMB

Figure 3 shows the mean peak angular velocities for elbow supination and pronation (Figures 3A,C), little MCP flexion (Figure 3B), and thumb internal rotation (Figure 3D) across participants in the two groups at different tempi. A two-way ANOVA with repeated measures found a group effect on all of these variables (Table 2), confirming greater elbow velocity for the professionals compared with the amateurs and greater velocities of the thumb and little 

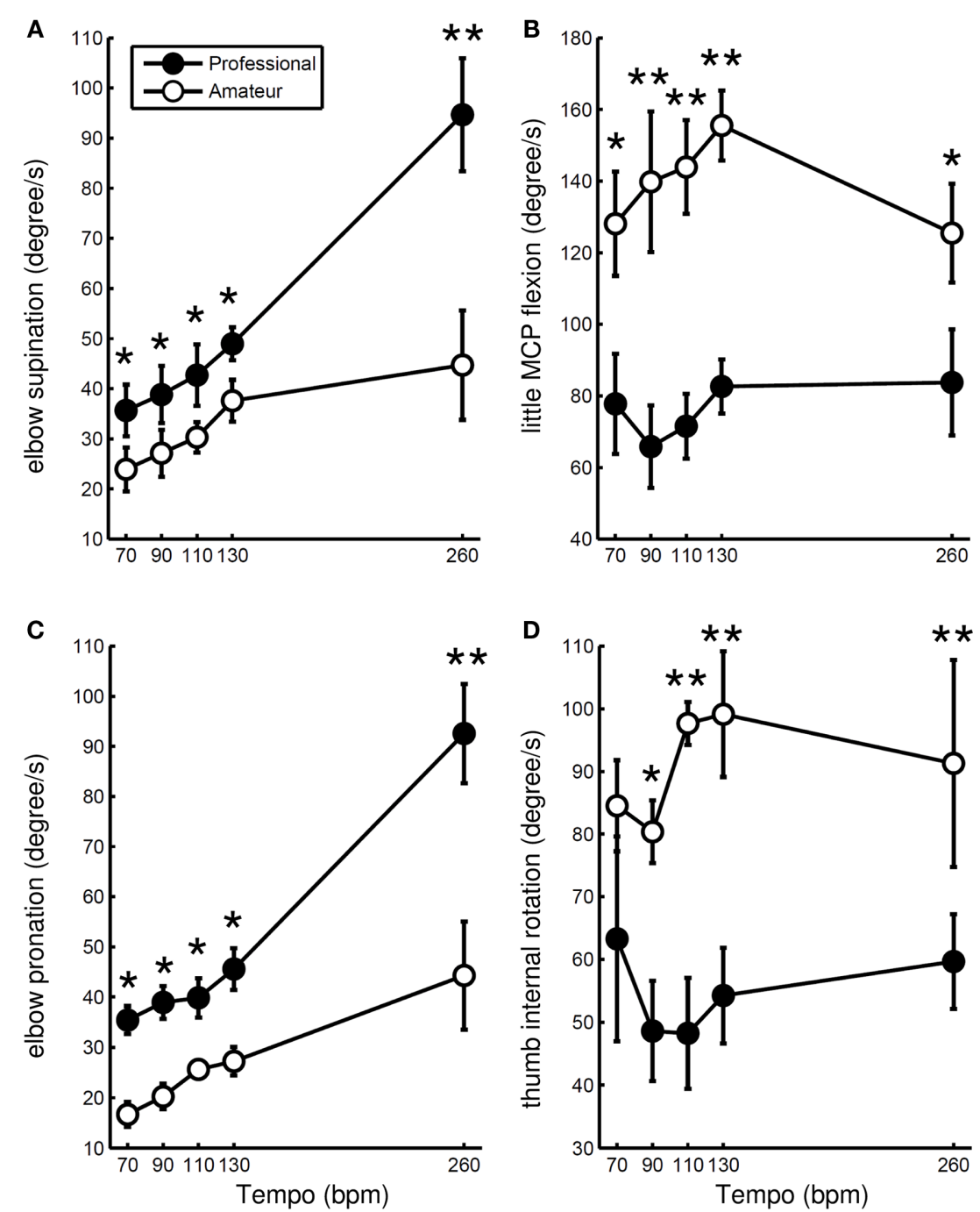

FIGURE 3 | Group means of peak angular velocities for elbow supination (A) and elbow pronation (C), little finger flexion (B), and thumb internal rotation (D) at five striking tempi for professional (filled circle) and amateur (open circle) pianists. Error bars represent $\pm 1 \mathrm{SE}(n=5) .{ }^{*} p<0.05,{ }^{* *} p<0.01$.

finger for the amateurs compared to the professionals. For the peak elbow supination and pronation velocity, both the effect of group $\mathrm{x}$ tempo interaction and the main effect of tempo were confirmed. The interaction effect indicated a greater increase in elbow longitudinal rotational velocity with increasing tempo for the professionals compared with the amateurs.

\section{TIME AT PEAK VELOCITY RELATIVE TO FINGER-KEY CONTACT MOMENT}

Table 3 shows the mean time at peak angular velocities for elbow supination and pronation (Figures 3A,C), little MCP flexion (Figure 3B), and thumb internal rotation (Figure 3D) relative to the moment of the corresponding finger-key contact across participants in the two groups at different tempi. The velocity reached its peak at approximately 50-70 ms following the moment of fingerkey contact at a slow tempo. However, as the tempo increased, the moment of peak velocity was earlier for both groups. This finding indicates that the duration of accelerating key descent with the fingertip became shorter in proportion to the tempo. Two-way repeated measures ANOVA demonstrated a main effect of tempo on all of these variables (Table 2). Neither a group $\mathrm{x}$ tempo interaction effect nor a main group effect was found.

To determine whether an earlier occurrence of peak joint angular velocity at a faster tempo resulted in an earlier occurrence of the peak linear descending velocity of the key, group means of the time of peak descending velocity of the key struck by the thumb and little finger relative to the moment of the corresponding finger-key contact across participants was further computed at different tempi. For the key struck by the thumb, the time at peak velocity at 70, $90,110,130$, and $260 \mathrm{bpm}$ was $79.3 \pm 7.3,83.9 \pm 5.7,75.0 \pm 3.8$, $72.0 \pm 7.6$, and $57.2 \pm 5.9 \mathrm{~ms}$, respectively, for the professionals and $83.7 \pm 1.9,78.6 \pm 0.9,75.2 \pm 0.4,70.9 \pm 2.1$, and $56.7 \pm 5.6 \mathrm{~ms}$, respectively, for the amateurs. Both of these players displayed a systematic decrease in the time of peak key velocity with increasing tempi. Similarly, for the key struck by the little finger, the time at peak velocity at $70,90,110,130$, and $260 \mathrm{bpm}$ was $76.6 \pm 8.5$, $82.1 \pm 8.2,75.7 \pm 2.0,72.9 \pm 6.3$, and $54.9 \pm 4.4 \mathrm{~ms}$, respectively, for 
the professionals and $79.3 \pm 4.2,77.2 \pm 2.2,75.5 \pm 1.9,72.2 \pm 2.1$, and $51.5 \pm 6.5 \mathrm{~ms}$, respectively, for the amateurs. For both variables, the tempo effect was significant $(p<0.01)$, confirming the earlier occurrence of peak key-descending velocity at faster tempi. This indicated that the fingertip accelerated to depress the key for a shorter duration with an increase in tempo.

\section{JOINT ANGLES OF THE NON-STRIKING FINGERS}

Figure 4 shows the mean joint angle at the MCP and PIP joints of the non-striking fingers (index, middle, and ring fingers) during keystrokes across participants in the two groups at different tempi. ANOVA confirmed a group effect only on the MCP joint at the

Table 2 | Results of two-way ANOVA with repeated measures.

\begin{tabular}{|c|c|c|c|}
\hline Variable & $\begin{array}{l}\text { Group } \\
F^{(1,8)}\end{array}$ & $\begin{array}{l}\text { Tempo } \\
\boldsymbol{F}^{(4,32)}\end{array}$ & $\begin{array}{l}\text { Group } \times \text { Tempo } \\
F^{(4,32)}\end{array}$ \\
\hline \multicolumn{4}{|l|}{ PEAK VELOCITY } \\
\hline Elbow supination & $11.74 * *$ & $11.93^{* *}$ & $3.38^{*}$ \\
\hline Elbow pronation & $36.38 * *$ & $17.50^{* *}$ & $2.90^{*}$ \\
\hline Little finger & $19.49 * *$ & 0.68 & 0.84 \\
\hline Thumb & $9.58^{*}$ & 0.72 & 1.00 \\
\hline \multicolumn{4}{|c|}{ TIME AT PEAK VELOCITY } \\
\hline Elbow supination & 0.48 & $51.68^{* *}$ & 1.39 \\
\hline Elbow pronation & 1.22 & $82.96 * *$ & 0.71 \\
\hline Little finger & 0.59 & $30.80 * *$ & 2.00 \\
\hline Thumb & 0.72 & $295.70^{* *}$ & 0.97 \\
\hline \multicolumn{4}{|c|}{ MEAN JOINT ANGLE } \\
\hline Index MCP & $8.26^{*}$ & 0.74 & 0.66 \\
\hline Middle MCP & $6.16^{*}$ & $3.66^{*}$ & 2.64 \\
\hline Ring MCP & 0.90 & 1.02 & 0.41 \\
\hline Index PIP & 0.08 & 1.57 & 0.38 \\
\hline Middle PIP & 0.11 & 1.34 & 0.67 \\
\hline Ring PIP & 0.23 & 1.37 & 1.29 \\
\hline \multicolumn{4}{|c|}{ MEAN EMG ACTIVITY } \\
\hline FDS & $36.16^{* *}$ & $34.40 * *$ & $20.68 * *$ \\
\hline EDC & $6.51 *$ & $16.42 * *$ & $10.68^{* *}$ \\
\hline Co-activation index & $6.70^{*}$ & $5.31 * *$ & $4.01 * *$ \\
\hline
\end{tabular}

${ }^{*} p<0.05,{ }^{*} p<0.01$ index and middle fingers (Table 2), which confirmed a smaller extension angle at the index and middle MCP joints for the professionals compared with the amateurs. There was no effect of tempo at any joint except for the middle MCP joint, where only amateurs had a slight increase in the angle with increasing tempo.

\section{MUSCULAR ACTIVITY}

Figure 5A illustrates muscular activity profiles at the EDC (positive) and FDS (negative) muscles across different tempi (70, 130, and $260 \mathrm{bpm}$ ) for one representative professional and one representative amateur pianist. Overall, these players showed increases in the activities of both muscles prior to the moment of finger-key contact, exhibiting their co-activation. In addition, the activity increased as the tempo increased. The professional player showed a smaller peak of activity for both muscles compared with the amateur for all tempi. The professional also exhibited smaller tonic activity, particularly at the EDC muscles, than the amateur.

Figures 5B-D show the average values of the mean activity at the FDS and EDC muscles and the CI values for these muscles during keystrokes across participants in the two groups at different tempi. There was a significant interaction effect of group and tempo on both the mean muscular activity of these two muscles and the CI value (Table 2). The interaction effect indicates a greater increase with tempo for the amateurs compared to the professionals. The effect of both group and tempo was also confirmed for all of these variables. The group effect indicates a greater muscular activity for the amateurs than for the professionals, whereas the tempo effect indicates an increase in muscular activity with tempo.

\section{INTRA-TRIAL CORRELATION BETWEEN RELATIVE TIMING ERROR AND MOVEMENT CHARACTERISTICS}

To assess if the deceleration of joint rotation due to the finger-key contact was correlated with the timing accuracy of the subsequent keystroke, a correlation between the peak angular deceleration (how much joint rotations at the elbow and digits were decelerated at the moment of collision) and relative timing error of the subsequent IKI was computed within a trial. The relative timing error was computed as follows: $\left(\mathrm{IKI}_{\text {exp }}-\mathrm{IKI}_{\text {obs }}\right) / \mathrm{IKI}_{\text {exp }}$. IKI $\mathrm{Ixp}_{\text {exp }}$ and IKI $\mathrm{obs}_{\text {o }}$ indicates the expected and observed IKI, respectively (Goebl and Palmer, 2008). The peak deceleration of joint rotation was determined within

Table 3 | Group means of the time at peak joint angular velocities at five striking tempi.

\begin{tabular}{|c|c|c|c|c|c|c|}
\hline & & 70 bpm & $90 \mathrm{bpm}$ & $110 \mathrm{bpm}$ & $130 \mathrm{bpm}$ & $260 \mathrm{bpm}$ \\
\hline \multirow[t]{2}{*}{ Elbow supination } & Professional & $46.6(2.0)$ & $36.0(1.7)$ & $35.0(10.4)$ & $29.8(6.1)$ & 19.4 (3.5) \\
\hline & Amatuer & $50.3(4.9)$ & $37.0(4.8)$ & $30.5(3.2)$ & $28.8(7.1)$ & $14.2(3.5)$ \\
\hline Elbor pronation & Amatuer & $57.3(8.3)$ & $51.9(8.1)$ & $42.6(5.9)$ & $39.8(5.3)$ & $12.9(3.8)$ \\
\hline Little finger & Professional & $74.1(10.4)$ & $57.4(8.0)$ & $38.6(15.7)$ & $40.6(8.9)$ & $13.6(8.6)$ \\
\hline Thumb & Amatuer & $63.8(4.3)$ & $53.9(3.6)$ & $48.0(1.5)$ & $41.2(4.1)$ & $16.4(1.9)$ \\
\hline
\end{tabular}

Time zero indicates the moment of the corresponding finger-key contact. (i.e., Little finger keypress for the peak vel. of little finger flexion and elbow supination, and thumb keypress for the peak vel. of thumb internal rotation and elbow pronation). Each number in parentheses indicates 1 SD across participants. 


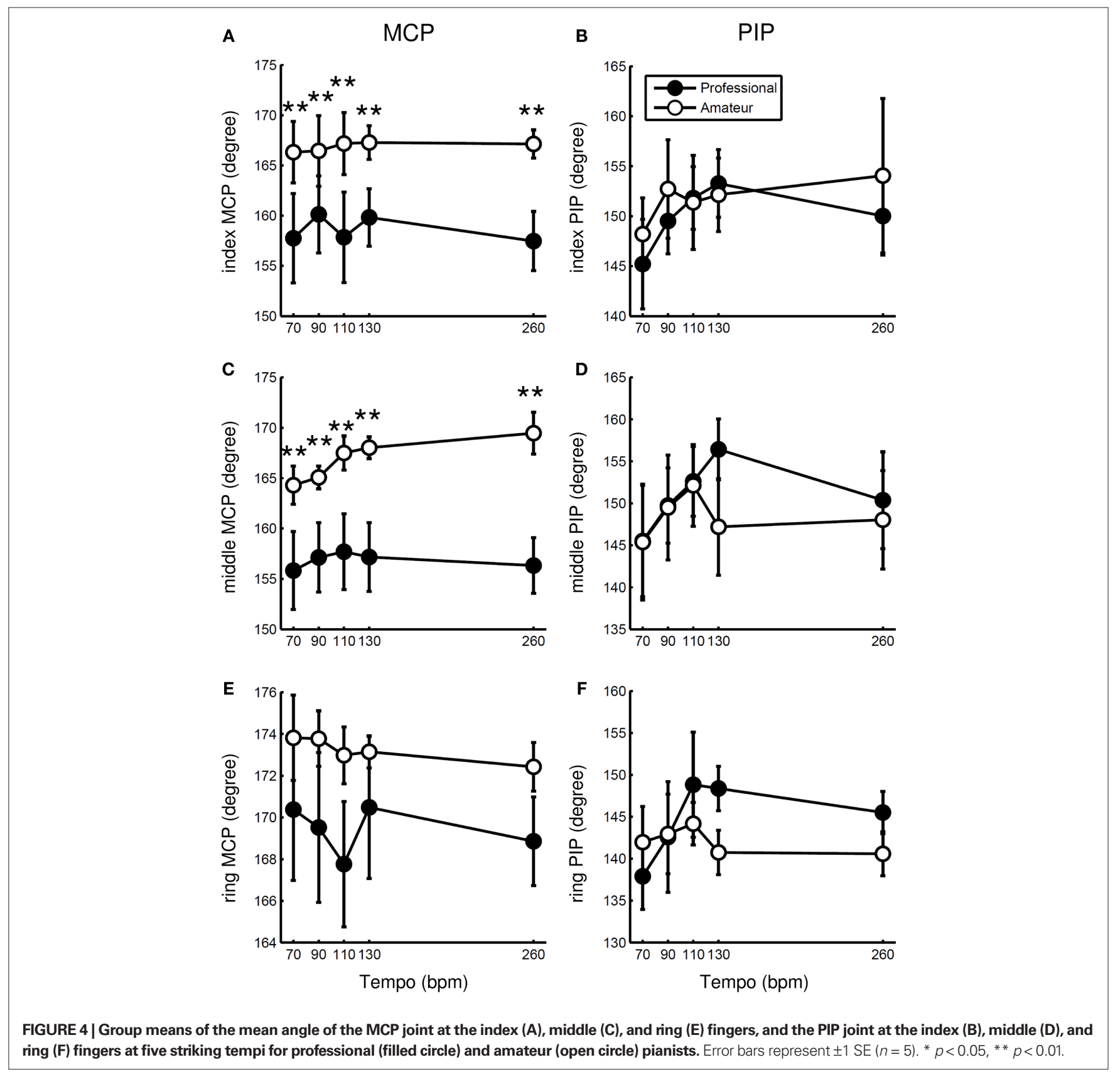

the range from -30 to $20 \mathrm{~ms}$ (Time zero indicates the moment of finger-key contact). Data from all five tempi was combined for the correlation analysis. Some keystrokes particularly at slower tempi showed no apparent peak of joint deceleration, which were not included for the analysis.

During the thumb keystroke, a significantly negative correlation was found for three of the professionals $(r=-0.26 \pm 0.37$; mean \pm SD across five players) and one of the amateurs $(r=0.12 \pm 0.38)$ for the elbow pronation, and for one of the professionals $(r=0.24 \pm 0.37)$ and two of the amateurs $(r=-0.12 \pm 0.37)$ for the thumb internal rotation. During the little finger keystroke, a negative correlation was evident for two of the professionals $(r=-0.03 \pm 0.36)$ and two of the amateurs $(r=-0.17 \pm 0.24)$ for the elbow supination, and for two of the professionals $(r=-0.06 \pm 0.37)$ and two of the amateurs $(r=-0.11 \pm 0.21)$ for the little MCP flexion. These findings indicate that some of the pianists performed more accurately timed keystrokes following the occurrence of stronger joint deceleration elicited by the finger-key contact, which suggests that proprioceptive feedback from muscle spindles ensures temporal accuracy of successive keystrokes. This view is in agreement with the report by Goebl and Palmer (2008).

\section{CORRELATION BETWEEN FASTEST TEMPO AND MOVEMENT CHARACTERISTICS}

To determine the movement features that account for individual differences in the fastest key-striking tempo across players, we initially computed the IKI during the fastest keystrokes. 

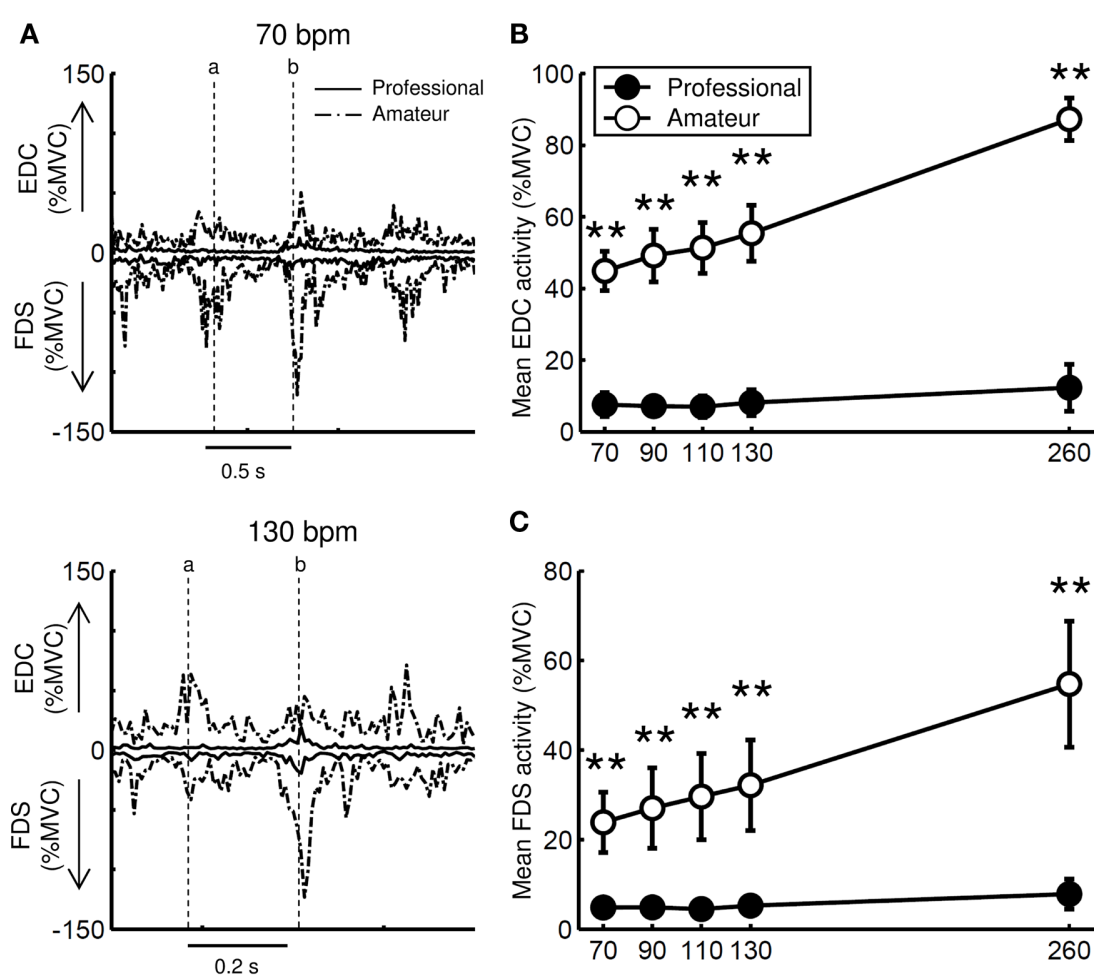

C
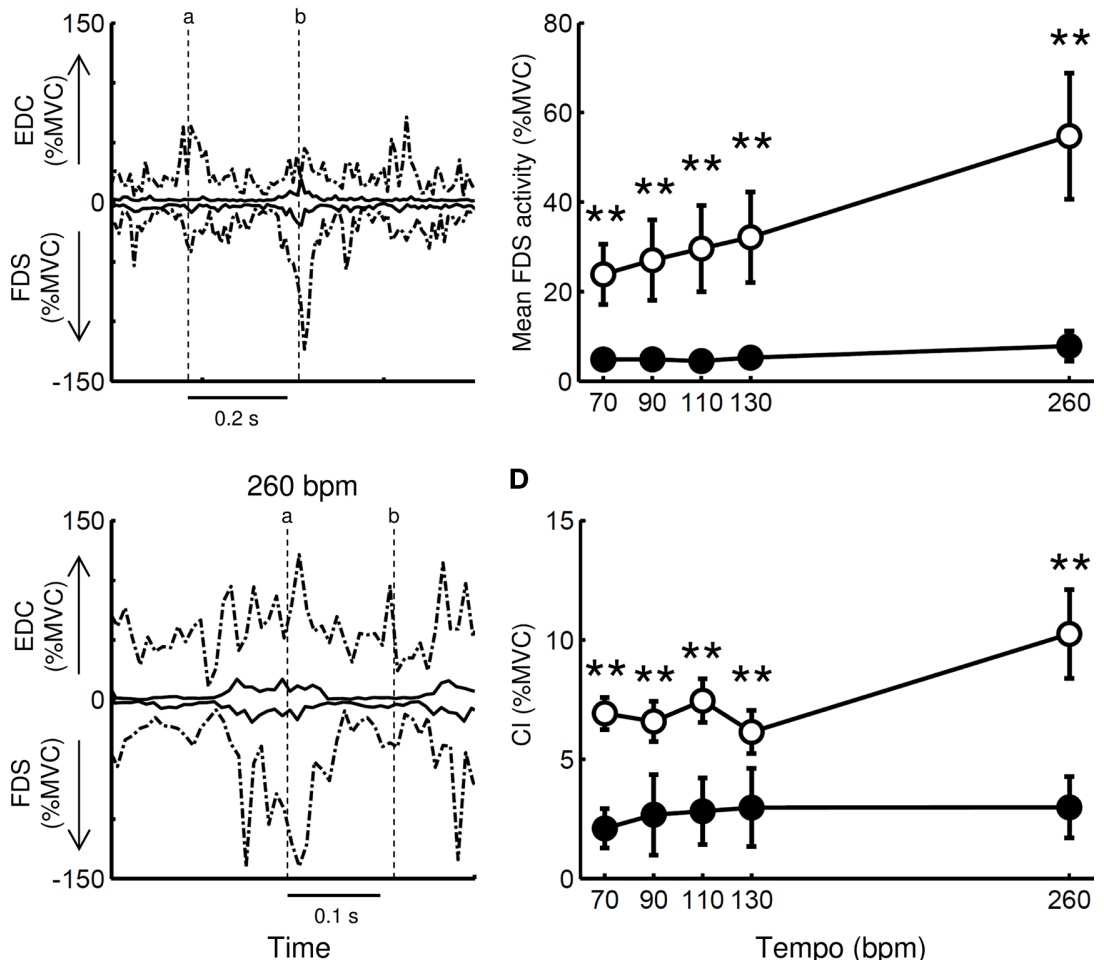

D

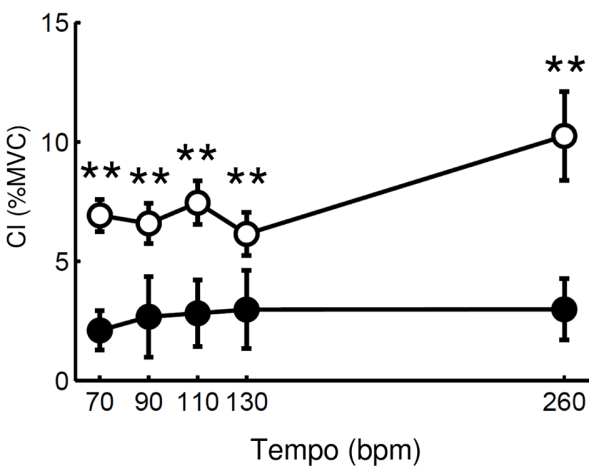

FIGURE 5 | (A) Time-history curves of muscular activity at the EDC (+) and FDS (-) across different tempi (70, 130, and $260 \mathrm{bpm})$ for a single trial. The solid and dashed lines represent one representative professional and one representative amateur pianist, respectively. A vertical dotted line denotes the moment when the key contacts the little finger (a) and the thumb (b). (B-D) Group means of the mean activity at the EDC (B) and FDS (C) muscles and the co-activation index (Cl) of these muscles (D) at five striking tempi for professional (filled circle) and amateur (open circle) pianists. Error bars represent $\pm 1 \mathrm{SE}(n=5) .{ }^{*} p<0.05,{ }^{*} p<0.01$.
The results for the professionals and amateurs were $156.6 \pm 8.6$ and $220.9 \pm 13.5 \mathrm{~ms}$, respectively, for the thumb keystrokes and $162.6 \pm 8.6$ and $220.8 \pm 12.8 \mathrm{~ms}$, respectively, for the little finger keystrokes (mean \pm SD across players). A $t$-test confirmed a significant group difference in each of the two variables $(p<0.01)$, indicating a faster keystroke rate for pianists with superior skill.

Using the dataset of the fastest tempo for all 10 participants, we performed a correlation analysis between the IKI (i.e., the mean value between the keystrokes of the thumb and little finger) and several fundamental movement variables. These variables included (1) the peak velocity for elbow longitudinal rotation, which is the average of the peak velocities between elbow pronation and supination, (2) the peak velocity for digit flexion, which is the average of the peak velocities between the thumb internal rotation and little MCP flexion, (3) the time at the peak descending velocity of the key relative to the moment of the keystroke, which is the mean between the two keys struck by the thumb and the little finger, and (4) the CI value. To minimize multiple statistical tests, we did not use the mean EMG activity and time at peak angular velocity of the elbow, thumb, and little fingers for the analysis because these variables should be largely related to the evaluated variables (i.e., the CI value and time at peak key descending velocity).

Table 4 summarizes the results of the correlation analysis. Based on a Bonferroni correction for multiple tests, we set a significant $p$-value of 0.0125 . We found significant correlations between the IKI at the fastest tempo of the 10 players and both 
Table 4 | Results of correlation analysis between the inter-keystroke interval at the fastest tempo and the movement variables for all players.

\begin{tabular}{|c|c|c|c|c|c|}
\hline \multirow[t]{4}{*}{ Variable } & & & \multicolumn{3}{|c|}{ Bootstrapping } \\
\hline & & & $95 \%$ co & fidence & \multirow[b]{3}{*}{$p$} \\
\hline & \multicolumn{2}{|c|}{ Correlation } & \multicolumn{2}{|c|}{ limit } & \\
\hline & $r$ & $p$ & Lower & Upper & \\
\hline Peak elbow rotational velocity & -0.75 & $0.012^{*}$ & -0.98 & -0.31 & $0.002^{*}$ \\
\hline Peak digit rotational velocity & 0.64 & 0.048 & -0.23 & 0.86 & 0.061 \\
\hline Time at peak key velocity & 0.86 & $0.001^{*}$ & 0.58 & 0.95 & $0.001 *$ \\
\hline Coactivation index $(\mathrm{Cl})$ & 0.60 & 0.064 & -0.32 & 0.86 & 0.039 \\
\hline
\end{tabular}

$r$ : correlation coefficient, $p$ : $p$-value ${ }^{*} p<0.0125$ with Bonferonni correction.

the peak elbow velocity and the time of peak key velocity. The negative and positive coefficients for the elbow velocity and the time at peak key velocity indicate a greater elbow velocity and earlier occurrence of peak key velocity for players who strike faster, respectively.

Having only used 10 datasets in the analysis, we acknowledge that outliers have potential to substantially influence the results. In order to further increase our confidence in the reliability of the results, we performed (1) a robust regression analysis (Hampel et al., 2005) and (2) a bootstrap procedure (Efron, 1979). The robust regression analysis evaluates if the gain significantly differs from zero. The result showed that the $p$-value for the peak elbow longitudinal rotational velocity, peak digit flexion velocity, time at the peak key velocity, and CI was $0.012,0.053,0.002$, and 0.077 , respectively. This verified the robustness of the results derived from the correlation analysis against outliers. The bootstrap procedure was also performed to assess if the correlation coefficient differed from zero. Table 4 (right) shows the 95th percentile confidence limits of the correlation coefficient derived from 1000 bootstrap samples and $p$-value indicating if the coefficient is significantly larger or smaller than zero. Both the upper and lower confidence limits were negative for the peak elbow velocity, and positive for the peak elbow velocity. The correlation coefficient for the peak elbow velocity and the time at the peak key velocity was significantly smaller and larger than zero, respectively.

To assess if particular kinematic variables are associated with the temporal precision of keystrokes during playing at the fastest tempo, we also performed the correlation analysis between the movement variables and coefficient of variance (CV: $\mathrm{SD} /$ mean) of the IKI within a trial. None of the movement variables showed a significant correlation, which was also confirmed by the robust regression analysis $(p>0.05)$.

\section{DISCUSSION}

\section{INTER-JOINT COORDINATION OF HAND AND FOREARM MOVEMENTS}

We found that professional pianists produced smaller flexion velocity at the thumb and little finger and greater elbow pronation and supination velocity during alternating keystrokes compared with amateurs. This finding indicated that pianists with superior skill used proximal limb motion to a greater extent to strike keys. In agreement with this finding, we had previously found that professional pianists took greater advantage of shoulder joint rotation than did novice players during a keystroke (Furuya and Kinoshita, 2008a,b). This movement allowed them to utilize the inter-segmental dynamics to move the elbow and wrist joints. However, the inter-segmental dynamics that propelled the finger movements were negligible. Therefore, it seems unlikely that, in this study, the pronounced rotation at the proximal joint in the professionals played a role in generating the inter-segmental dynamics that effectively drive finger motion. This view is strengthened by the concurrence of peak velocities at the elbow and digits (Figure 4), which contrasts with previous findings of the occurrence of peak joint velocity in the order from proximal to distal when utilizing inter-segmental dynamics (Dounskaia et al., 1998; Buchanan, 2004; Furuya and Kinoshita, 2007). We speculate that elbow rotation directly contributes to the production of fingertip descending velocity to strike a key. A similar motor skill was also reported for a ball-throwing motion, where skilled throwers took greater advantage of the shoulder's internal rotation to accelerate the hand motion just prior to releasing the ball compared to unskilled individuals (Hore et al., 2005; Gray et al., 2006).

There are at least two benefits of taking advantage of the proximal joint motion. First, the distance between the joint center and the endpoint of the linked system is longer for more proximal joints. Therefore, joint rotation around more proximal joint results in a larger translational velocity at the limb endpoint, which provides proximal joint rotation with mechanical advantage. Second, proximal muscles have greater physiological cross-sectional areas than distal muscles. Because the tolerance to muscular fatigue increases in proportion to the cross-sectional area (Herzog, 2000), utilization of proximal joint motion could ensure fast and accurate movements for longer periods of time. Indeed, we found smaller loads on extrinsic finger muscles for the professionals compared to the amateurs. Presumably, extensive piano training involving extraordinary repetitive strokes could allow pianists to acquire inter-joint coordination that has mechanical and physiological advantages. This perspective is in agreement with Bernstein's hypothesis regarding the use of a greater number of DOFs to economize movement production with improvements in motor skills (Bernstein, 1967). Furthermore, observations in favor of Bernstein's hypothesis in both discrete (Furuya and Kinoshita, 2008a) and cyclic motor behaviors, in spite of their distinct differences in neural control mechanisms (Schaal et al., 2004), suggest that using a greater number of DOFs during skill improvement might be a common principle governing skill acquisition in multi-joint movements.

\section{ORGANIZATION OF HAND POSTURE}

Another key finding of the present study was smaller extension angles at the fingers that were not used for striking keys for the professionals compared with the amateurs. The muscular activities of finger extensors that are responsible for lifting the fingers were also smaller for the professionals than for the amateurs. These results indicate that the professionals reduced the muscular load for keeping the non-striking fingers lifted during the course of the keystrokes. Because a systematic increase in the extension angle of non-striking fingers with tempo was not necessarily observed, the role of this postural muscular contraction is unlikely to compensate for an unwanted spillover effect of the striking motion on 
the non-striking fingers due to anatomical and neural connections across digits (Hager-Ross and Schieber, 2000; Lang and Schieber, 2004; Aoki et al., 2005; van Duinen et al., 2009; Yu et al., 2010). Instead, this finger elevation plays a role in simply avoiding sounding unwanted tones.

The hand is a highly redundant motor system with a large number of DOFs. Previous studies have demonstrated that the organization of hand posture during a manual grasping task is subject to task constraints, such as position of finger location to be placed on the object (Lukos et al., 2007), the geometric shape of the object (Santello et al., 1998), and the movement speed during reaching toward the object (Rand et al., 2006). However, whether hand posture would differ depending on the skill of the individual has remained unclear. In the present motor task, although the explicit constraint imposed on non-striking fingers was only to lift the hand to avoid touching unwanted keys, there was a distinct difference in hand posture between the professional and amateur pianists. This finding implies that the nervous system reorganizes hand posture during keystrokes with an improvement in skill. There seem at least two feasible benefits of this reorganization of hand posture. First, hand posture with smaller load at finger muscles would facilitate endurance to peripheral muscular fatigue, which is a concern for piano playing (Penn et al., 1999). Second, smaller force exertion at finger muscles could reduce muscular stiffness, which decreases mechanical constraints limiting independent control of finger movements (Leijnse, 1997; Lang and Schieber, 2004). We therefore inferred that pianists with superior skill took account of maximizing endurance to muscular fatigue and/or independent control of digits when organizing the hand posture during repetitive keystrokes.

\section{MOVEMENT STRATEGY FOR STRIKING KEYS OVER A WIDE RANGE OF TEMPI}

As the tempo increased, the rotational velocity of the elbow, thumb, and little finger reached its peak earlier. This finding indicated that the fingertip accelerated to depress the key for a shorter duration with an increase in tempo. Nevertheless, the key's descending velocity did not decrease at a faster tempo. This finding can be explained in terms of increases in both elbow velocity and muscular co-contraction with increasing tempo. These increases should allow for a greater transfer of momentum from the limb to the key while the fingertip is colliding with the key. It is therefore likely that both the professional and the amateur players took greater advantage of momentum transfer as the tempo increased to compensate for the failure to fully accelerate key depression. Indeed, pianists produced a fingertip velocity that was larger than the key-descending velocity only during a keystroke with a touch that was able to utilize momentum transfer (Furuya et al., 2010).

Intriguingly, we also found an interaction effect of group and tempo on the peak elbow velocity and on the finger muscular coactivation level. The professionals showed a greater tempo-related increase in elbow velocity than did the amateurs, an effect being reversed for co-activation. This finding suggests that the professionals increased the descending velocity at the fingertip to a greater extent and the stiffness at the fingertip to a lesser extent when striking faster compared to the amateurs, which highlights a skill level-dependent difference in the movement strategy to adjust tempo. Furthermore, when striking at the fastest tempo, a distinct inter-pianist difference in the IKI was clearly explained by both the elbow longitudinal rotational velocity and the time at peak fingertip descending velocity. This finding supported our view that a failure to fully accelerate the key depression with the fingertip when striking faster was compensated for by elbow motion, which reflects a player's expertise. Taken together, the distinct movement strategy for tempo adjustment in pianists with superior skill may play a role in the execution of extremely fast keystrokes. One implication of this finding for music pedagogy would be to produce motion at proximal joints but not distal joints to strike keys faster, which might be counterintuitive for less skilled players.

Previous neuroimaging studies have demonstrated that pianists with superior skill had a greater volume of gray matter in the motor cortex (Amunts et al., 1997) and cerebellum (Hutchinson et al., 2003). Enlargement of these motor-related brain regions, which can result from long-term training from childhood (Zatorre et al., 2007; Penhune, 2011), has been mostly explained in terms of superior hand motor function, such as faster speed of finger tapping (Amunts et al., 1997). However, both the primary motor cortex (Vargas-Irwin et al., 2010) and the cerebellum (Thach, 1998; Timmann et al., 2008) play roles in coordinating multiple DOFs. Our findings therefore raise the possibility that a greater volume of motor-related regions may enable pianists to utilize more DOFs, particularly at the proximal body portion, to perform virtuosic motor behaviors. This supposition was compatible with a theory proposing that as motor skill develops, DOFs that were initially redundant become abundant so as to enhance movement performance (Yang and Scholz, 2005; Latash, 2008). Such a change may be associated with an enlargement of motor-related brain regions with an improvement of motor skill for piano playing.

The present findings may provide insights into motor control of piano touch. Previous studies demonstrated that touch in piano keystroke was defined by distinct mechanical interaction between the fingertip and key (Goebl et al., 2005; Kinoshita et al., 2007; Goebl and Palmer, 2008). Our study also suggested different finger-key contact dynamics between the professionals and amateurs particularly at faster tempi, because the pianists with superior skill showed a smaller increase in the finger muscular co-activation and thus stiffness with tempo. However, the smaller increase in the co-activation was likely attributed to a greater increase in elbow velocity with tempo. The utilization of the elbow motion for the professionals thus allowed for the fingertip-key contact with low stiffness over a wide range of tempi. This implicates that the proximal joint motion is a key determinant of piano touch, which supports our recent finding (Furuya et al., 2010).

\section{SPECIALIZED MOTOR SKILL RESPONSIBLE FOR ARTISTIC MUSICAL PERFORMANCE}

To manipulate elements of music (e.g., rhythm, timbre, loudness, harmony, and tempo) elicits emotional and autonomic responses during listening to music (Dalla Bella et al., 2001; Gomez and Danuser, 2007; Bernardi et al., 2009; Hailstone et al., 2009). Artistic musical performance thus requires motor skill to manipulate elements of music. The present study focused on motor skill to change tempo, a key variable affecting listeners' emotion and autonomic activity (Dalla Bella et al., 2001; Khalfa et al., 2008). We identified 
movement characteristics responsible for tempo adjustment during alternate piano keystrokes, which however differed depending on players' expertise. Remarkably, the differences appeared to enable pianists with superior skill to perform faster keystrokes, which allowed for a wider range of musical expression. In addition, a less pronounced increase in finger muscular activity with tempo for the professional pianists than the amateurs could help in individuated finger movements and/or prevention of muscular fatigue, both of which are necessary for precise tone production. Accordingly, the specialized motor skill to manipulate tempo for the professional pianists would ensure artistic musical performance with superior expressiveness and precision.

\section{CONCLUSION}

The present study determined certain motor skills responsible for artistic musical performance. To maintain fine motor performance for a prolonged duration of repetitive keystrokes, the professional pianists had faster elbow rotation, slower rotation at the digits used for keystrokes, smaller extension angle at the fingers unused for keystrokes, and smaller co-activation of finger muscles compared with

\section{REFERENCES}

Amunts, K., Schlaug, G., Jäncke, L., Steinmetz, H., Schleicher, A., Dabringhaus, A., and Zilles, K. (1997). Motor cortex and hand motor skills: structural compliance in the human brain. Hum. Brain Mapp. 5, 206-215.

Aoki, T., Furuya, S., and Kinoshita, H. (2005). Finger-tapping ability in male and female pianists and nonmusician controls. Motor. Control. 9, 23-39.

Bengtsson, S. L., Nagy, Z., Skare, S., Forsman, L., Forssberg, H., and Ullen, F. (2005). Extensive piano practicing has regionally specific effects on white matter development. Nat. Neurosci. 8, 1148-1150.

Bernardi, L., Porta, C., Casucci, G., Balsamo, R., Bernardi, N. F., Fogari, R., and Sleight, P. (2009). Dynamic interactions between musical, cardiovascular, and cerebral rhythms in humans. Circulation 119, 3171-3180.

Bernstein, N. (1967). The Coordination and Regulation of Movements. Oxford: Pergamon Press.

Buchanan, J. J. (2004). Learning a single limb multijoint coordination pattern: the impact of a mechanical constraint on the coordination dynamics of learning and transfer. Exp. Brain Res. 156, 39-54.

Dalla Bella, S., Peretz, I., Rousseau, L., and Gosselin, N. (2001). A developmental study of the affective value of tempo and mode in music. Cognition 80, B1-B10.

Dounskaia, N. (2010). Control of human limb movements: the leading joint hypothesis and its practical applications. Exerc. Sport Sci. Rev. 38, 201-208.
Dounskaia, N. V., Swinnen, S. P., Walter, C. B., Spaepen, A. J., and Verschueren, S. M. (1998). Hierarchical control of different elbow-wrist coordination patterns. Exp. Brain Res. 121, 239-254.

Efron, B. (1979). Bootstrap methods: another look at the jackknife. Ann. Stat. 7, 1-26.

Feltner, M., and Taylor, G. (1997). Threedimensional kinetics of the shoulder, elbow, and wrist during a penalty throw in water polo. J. Appl. Biomech. 13, 347-372.

Fujii, S., Kudo, K., Ohtsuki, T., and Oda S. (2009a). Tapping performance and underlying wrist muscle activity of non-drummers, drummers, and the world's fastest drummer. Neurosci. Lett. 459, 69-73.

Fujii, S., Kudo, K., Shinya, M., Ohtsuki, T., and Oda, S. (2009b). Wrist muscle activity during rapid unimanual tapping with a drumstick in drummers and nondrummers. Motor. Control. 13, 237-250.

Furuya, S., Altenmüller, E., Katayose, H., and Kinoshita, H. (2010). Control of multi-joint arm movements for the manipulation of touch in keystroke by expert pianists. BMC Neurosci. 11, 82 .

Furuya, S., Aoki, T., Nakahara, H., and Kinoshita, H. (2011). Individual differences in the biomechanical effect of loudness and tempo on upperlimb movements during repetitive piano keystrokes. Hum. Mov. Sci. doi: 10.1016/j.humov.2011.01.002. [Epub ahead of print].

Furuya, S., and Kinoshita, H. (2007). Roles of proximal-to-distal sequential organization of the upper limb

the amateur pianists. The professionals also increased the elbow velocity to a greater extent and the co-activation to a smaller extent than the amateurs with an increase in tempo. Furthermore, during striking at the individual's fastest tempo, pianists capable of faster keystrokes showed greater elbow velocity and earlier occurrence of the peak key velocity, which highlights the importance of the specialized motor skill for a wider range of tempo manipulation. A future study is needed to assess if the findings can be generalized to melodic sequences involving motions at greater number of digits and less pronounced arm motion, and if the findings of individual differences can be generalized to a larger number of pianists.

\section{ACKNOWLEDGMENTS}

We thank Drs. Masaya Hirashima (Tokyo University) and Takashi Takuma (Osaka Institute of Technology) for their helpful and constructive suggestions on the calculation of joint angles based on the 3D position data of markers. We also thank Mr. Hrishikesh Rao (University of Minnesota) for proofreading the manuscript. We appreciate three anonymous reviewers who provided constructive suggestions to improve the manuscript.

segments in striking the keys by expert pianists. Neurosci. Lett. 421, 264-249.

Furuya, S., and Kinoshita, H. (2008a) Expertise-dependent modulation of muscular and non-muscular torques in multi-joint arm movements during piano keystroke. Neuroscience 156 390-402.

Furuya, S., and Kinoshita, H. (2008b). Organization of the upper limb movement for piano key-depression differs between expert pianists and novice players. Exp. Brain Res. 185, 581-593.

Furuya, S., Osu, R., and Kinoshita, H. (2009). Effective utilization of gravity during arm downswing in keystrokes by expert pianists. Neuroscience 164 , 822-831.

Furuya, S., and Soechting, J. F. (2010). Role of auditory feedback in the control of successive keystrokes during piano playing. Exp. Brain Res. 204, 223-237.

Goebl, W., Bresin, R., and Galembo, A (2005). Touch and temporal behavior of grand piano actions. J. Acoust. Soc. Am. 118, 1154-1165.

Goebl, W., and Palmer, C. (2008). Tactile feedback and timing accuracy in piano performance. Exp. Brain Res. 186, 471-479.

Gomez, P., and Danuser, B. (2007). Relationships between musical structure and psychophysiological measures of emotion. Emotion 7, 377-387.

Gray, S., Watts, S., Debicki, D., and Hore, J. (2006). Comparison of kinematics in skilled and unskilled arms of the same recreational baseball players. J. Sports Sci. 24, 1183-1194.

Hager-Ross, C., and Schieber, M. H. (2000). Quantifying the independence of human finger movements: comparisons of digits, hands, and movement frequencies. J. Neurosci. 20, 8542-8550.

Hailstone, J. C., Omar, R., Henley, S. M., Frost, C., Kenward, M. G., and Warren, J. D. (2009). It's not what you play, it's how you play it: timbre affects perception of emotion in music. Q. J. Exp. Psychol. (Colchester) 62, 2141-2155.

Hampel, F. R., Ronchetti,E.M., Rousseeuw, P. J., and Stahel, W. A. (2005). Robust Statistics: The Approach Based on Influence Functions. New York, NY: Wiley Series in Probability and Statistics.

Herzog, W. (2000). Muscle properties and coordination during voluntary movement. J. Sports Sci. 18, 141-152.

Hirashima, M., Kudo, K., Watarai, K., and Ohtsuki, T. (2007). Control of $3 \mathrm{D}$ limb dynamics in unconstrained overarm throws of different speeds performed by skilled baseball players. J. Neurophysiol. 97, 680-691.

Hogan, N., and Sternad, D. (2007). On rhythmic and discrete movements: reflections, definitions and implications for motor control. Exp. Brain Res. 181, 13-30.

Hore, J., Debicki, D. B., and Watts, S. (2005). Braking of elbow extension in fast overarm throws made by skilled and unskilled subjects. Exp. Brain Res. 164, 365-375.

Hutchinson, S., Lee, L. H., Gaab, N., and Schlaug, G. (2003). Cerebellar volume of musicians. Cereb. Cortex 13, 943-949.

Huys, R., Studenka, B. E., Rheaume, N. L. Zelaznik, H. N., and Jirsa, V.K. (2008). Distinct timing mechanisms produce 
discrete and continuous movements. PLoS Comput. Biol. 4, e1000061. doi: 10.1371/journal.pcbi.1000061

Jäncke, L., Schlaug, G., and Steinmetz, H. (1997). Hand skill asymmetry in professional musicians. Brain Cogn. 34, 424-432.

Kay, B. A., Turvey, M. T., and Meijer, O. G. (2003). An early oscillator model: studies on the biodynamics of the piano strike (Bernstein \& Popova, 1930). Motor. Control. 7, 1-45.

Kellis, E., Arabatzi, F., and Papadopoulos, C. (2003). Muscle co-activation around the knee in drop jumping using the co-contraction index. J. Electromyogr. Kinesiol. 13, 229-238.

Khalfa, S., Roy, M., Rainville, P., Dalla Bella, S., and Peretz, I. (2008). Role of tempo entrainment in psychophysiological differentiation of happy and sad music? Int. J. Psychophysiol. 68, 17-26.

Kinoshita, H., Furuya, S., Aoki, T., and Altenmuller, E. (2007). Loudness control in pianists as exemplified in keystroke force measurements on different touches. J. Acoust. Soc. Am. 121, 2959-2969.

Kugimoto, N., Miyazono, R., Omori, K., Fujimura, T., Furuya, S., Katayose, H., Miwa, H., and Nagata, N. (2009). CG Animation for Piano Performance. New York, NY: ACM, 1-1.

Lang, C. E., and Schieber, M. H. (2004). Human finger independence: limitations due to passive mechanical coupling versus active neuromuscular control. J. Neurophysiol. 92, 2802-2810.
Latash, M. L. (2008). Synergy. New York, NY: Oxford University Press.

Leijnse, J. N. (1997). Anatomical factors predisposing to focal dystonia in the musician's hand--principles, theoretical examples, clinical significance. J. Biomech. 30, 659-669.

Loehr, J. D., and Palmer, C. (2009). Subdividing the beat: auditory and motor contributions to synchronization. Music Percept. 26, 415-425.

Lukos, J., Ansuini, C., and Santello, M. (2007). Choice of contact points during multidigit grasping: effect of predictability of object center of mass location. J. Neurosci. 27, 3894-3903.

Meulenbroek, R. G., Rosenbaum, D. A., Thomassen, A. J., and Schomaker, L. R. (1993). Limb-segment selection in drawing behaviour. Q. J. Exp. Psychol. A. 46, 273-299.

Münte, T. F., Altenmüller, E., and Jäncke, L. (2002). The musician's brain as a model of neuroplasticity. Nat. Rev. Neurosci. 3, 473-478.

Palmer, C., Koopmans, E., Loehr, J. D., and Carter, C. (2009). Movement-related feedback and temporal accuracy in clarinet performance. Music Percept. 26, 439-449.

Parlitz, D., Peschel, T., and Altenmüller, E. (1998). Assessment of dynamic finger forces in pianists: effects of training and expertise. J. Biomech. 31, 1063-1067.

Penhune, V. B. (2011). Sensitive periods in human development: Evidence from musical training. Cortex, doi: 10.1016/j.cortex.2011.05.010. [Epub ahead of print].
Penn, I. W., Chuang, T. Y., Chan, R. C. and Hsu, T. C. (1999). EMG power spectrum analysis of first dorsal interosseous muscle in pianists. Med. Sci. Sports Exerc. 31, 1834-1838.

Pfann, K. D., Corcos, D. M., Moore, C. G., and Hasan, Z. (2002). Circle-drawing movements at different speeds: role of inertial anisotropy. J. Neurophysiol. 88 2399-2407.

Rand, M. K., Squire, L. M., and Stelmach, G.E. (2006). Effect of speed manipulation on the control of aperture closure during reach-to-grasp movements. Exp. Brain Res. 174, 74-85.

Santello, M., Flanders, M., and Soechting, J. F. (1998). Postural hand synergies for tool use. J. Neurosci. 18, 10105-10115.

Schaal, S., Sternad, D., Osu, R., and Kawato, M. (2004). Rhythmic arm movement is not discrete. Nat Neurosci. 7, 1136-1143.

Thach, W. T. (1998). A role for the cerebellum in learning movement coordination. Neurobiol. Learn. Mem. 70, 177-188.

Timmann, D., Lee, P., Watts, S., and Hore, J. (2008). Kinematics of arm joint rotations in cerebellar and unskilled subjects associated with the inability to throw fast. Cerebellum 7, 366-378.

van Duinen, H., Yu, W. S., and Gandevia, S. C. (2009). Limited ability to extend the digits of the human hand independently with extensor digitorum. $J$. Physiol. 587, 4799-4810.

Vargas-Irwin, C. E., Shakhnarovich, G. Yadollahpour, P., Mislow, J.M., Black, M. J., and Donoghue, J.P. (2010). Decoding complete reach and grasp actions from local primary motor cortex populations. J. Neurosci. 30, 9659-9669.

Yang, J. F., and Scholz, J. P. (2005). Learning a throwing task is associated with differential changes in the use of motor abundance. Exp. Brain Res. 163, 137-158.

Yu, W. S., van Duinen, H., and Gandevia, S. C. (2010). Limits to the control of the human thumb and fingers in flexion and extension. J.Neurophysiol. 103,278-289.

Zatorre, R. J., Chen, J. L., and Penhune, V. B. (2007). When the brain plays music: auditory-motor interactions in music perception and production. Nat. Rev. Neurosci. 8, 547-558.

Conflict of Interest Statement: The authors declare that the research was conducted in the absence of any commercial or financial relationships that could be construed as a potential conflict of interest.

Received: 27 March 2011; accepted: 12 May 2011; published online: 27 May 2011.

Citation: Furuya S, Goda T, Katayose H,

Miwa H and Nagata N (2011) Distinct inter-joint coordination during fast alternate keystrokes in pianists with superior skill. Front. Hum. Neurosci. 5:50. doi 10.3389/fnhum.2011.00050

Copyright ( $) 2011$ Furuya, Goda, Katayose, Miwa and Nagata. This is an open-access article subject to a non-exclusive license between the authors and Frontiers Media $S A$, which permits use, distribution and reproduction in other forums, provided the original authors and source are credited and other Frontiers conditions are complied with. 